Pneumologe 2017 · 14:129-130

DOI 10.1007/s10405-017-0114-1

Online publiziert: 8. Mai 2017

(c) Springer Medizin Verlag GmbH 2017

CrossMark

Unter dem Begriff Screening versteht man ein systematisches Testverfahren, das eingesetzt wird, um innerhalb eines definierten Prüfbereichs Elemente $\mathrm{zu}$ finden, die bestimmte Eigenschaften aufweisen. Mit dem Screening soll also etwas der Aufmerksamkeit zugeführt werden, das sonst im Verborgenen bleiben würde. Die Herausforderung beim Screening besteht darin, dass eine große Gruppe von scheinbar Gesunden untersucht werden muss, um wenige symptomlose Krankheitsträger zu identifizieren. Im deutschsprachigen Bereich werden derartige Maßnahmen auch mit „Früherkennung“ umschrieben, jedoch hat sich der Begriff "Screening“ auch hier zunehmend durchgesetzt.

Bei jedem Screening spielt es eine große Rolle, die Risikopopulation möglichst gut zu definieren. Betrachtet man z. B. das Lungenkrebsscreening, ist die Anwendung von Röntgenstrahlen im Rahmen der „Low-dose“-Computertomographie nur dann $\mathrm{zu}$ rechtfertigen, wenn eine Hochrisikogruppe, definiert über Alter und Raucherstatus, untersucht wird [1].

Das Lungenkarzinom ist nachweislich das Karzinom mit der höchsten Sterberate bei Mann und Frau [2]. Zudem sind die obstruktiven Atemwegserkrankungen mittlerweile anerkannte Volkskrankheiten [3] und auch andere Lungenerkrankungen, wie interstitielle Lungenerkrankungen, pulmonale $\mathrm{Hy}$ pertonie oder Mukoviszidose verlassen nach und nach den Bereich der seltenen Krankheiten.

Durch eine Vielzahl neuer therapeutischer Möglichkeiten, sei es medikamentös [4], interventionell [5] oder chirurgisch, bestehen Optionen, Patienten mit Lungenerkrankungen ein längeres und lebenswertes Leben zu ermöglichen.

\author{
F. J. F. Herth ${ }^{1} \cdot$ H. Olschewski ${ }^{2}$ \\ ${ }^{1}$ Thoraxklinik, Universitätsklinikum Heidelberg, Heidelberg, Deutschland \\ ${ }^{2}$ Klin. Abt. für Pulmonologie, Medizinische Universitätsklinik, Graz, Österreich
}

\title{
Screening in der Pneumologie
}

Hierzu müssten die potenziellen Patienten jedoch frühzeitiger erkannt werden. Nach wie vor gilt für viele pulmonale Erkrankungen, dass sie erst in einem fortgeschrittenen Erkrankungsstadium diagnostiziert werden. Dies bedeutet in der Regel nur mehr eingeschränkte Therapieoptionen, da bei den meisten dieser Erkrankungen nur eine wirklich frühzeitige Therapie eine dauerhafte Stabilisierung oder gar Heilung erlaubt.

In diesem Heft möchten wir die verschiedenen Optionen bei unterschiedlichen Lungenerkrankungen hinsichtlich Früherkennung bzw. Screening erläutern. Neben den großen Lungenerkrankungen wird auch ein Blick über den Tellerrand gewagt. Das Screening hinsichtlich Brustkrebs ist ein Verfahren, welches in Deutschland etabliert wurde und für das bereits umfangreiche Erfahrungen bestehen. Hier werden die Anfangsschwierigkeiten und ihre Lösungen, die Vorteile aber auch Nachteile präsentiert. Das mag richtungsweisend auch für die Etablierung von Verfahren zum Screening von Lungenkrankheiten sein.

\section{》) Viele pulmonale Erkrankun- gen lassen sich nur mit einer frühzeitigen Therapie dauerhaft verbessern}

Ob Screening wirklich Sinn macht, ist sicher eine Definitionsfrage und hängt davon ab, wie das Verfahren implementiert wird. Für viele zu screenende Erkrankungen gibt es keine soliden gesundheitsökonomischen Daten. Häufig müssen sehr viele Personen untersucht werden, bis einer davor profitiert. Aus Sicht des gescreenten Betroffenen mit einem dadurch deutlich verlängerten Überleben eröffnet sich selbstverständlich eine ganz andere Sichtweise. Zu bedenken gilt beim Screening im Bereich der Lungenkrankheiten, insbesondere beim Lungenkarzinom und bei obstruktiven Lungenerkrankungen, dass jedes Screening mit der Etablierung einer Raucherentwöhnung gekoppelt sein muss.

Wir hoffen, Ihnen mit diesem Heft zum Stellenwert der verschiedenen Screening-Modalitäten bei unterschiedlichen Erkrankungen einen interessanten Überblick zu bieten, der es Ihnen erlaubt, in Ihrem täglichen Alltag zu entscheiden, ob und mit welcher Methode mögliche Patienten untersucht werden sollten.

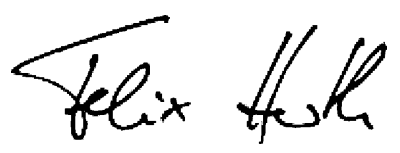

Prof. Dr. Felix Herth

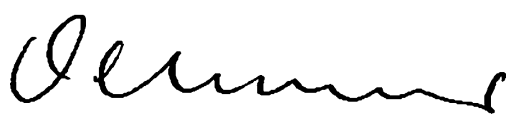

Prof. Dr. Horst Olschewski

\section{Korrespondenzadresse}

\section{Prof. Dr. F. J. F. Herth}

Thoraxklinik, Universitätsklinikum Heidelberg Röntgenstr. 1, 69126 Heidelberg, Deutschland felix.herth@med.uni-heidelberg.de

\section{Prof. Dr. H. Olschewski}

Klin. Abt. für Pulmonologie, Medizinische Universitätsklinik

Auenbruggerplatz 20, $8036 \mathrm{Graz}$, Österreich horst.olschewski@medunigraz.at

Interessenkonflikt. F.J.F. Herth und H. Olschewski geben an, dass kein Interessenkonflikt besteht. 


\section{Literatur}

1. National Lung Screening Trial Research et al (2011) Reduced lung-cancer mortality with low-dose computed tomographic screening. N Engl I Med 365(5):395-409

2. American Cancer Society (2017) Cancer facts \& figures. Atlanta, GA, USA. https://www.cancer.org/ research/cancer-facts-statistics/all-cancer-factsfigures/cancer-facts-figures-2017.html

3. Lopez-Campos JL, Tan W, Soriano JB (2016) Global burden of COPD. Respirology 21(1):14-23

4. Reck M et al (2016) Pembrolizumab versus chemotherapy for PD-L1-positive non-small-cell lung cancer. N Engl J Med 375(19):1823-1833

5. Herth FJ et al (2016) Endoscopic lung volume reduction: an expert panel recommendation. Respiration 91(3):241-250

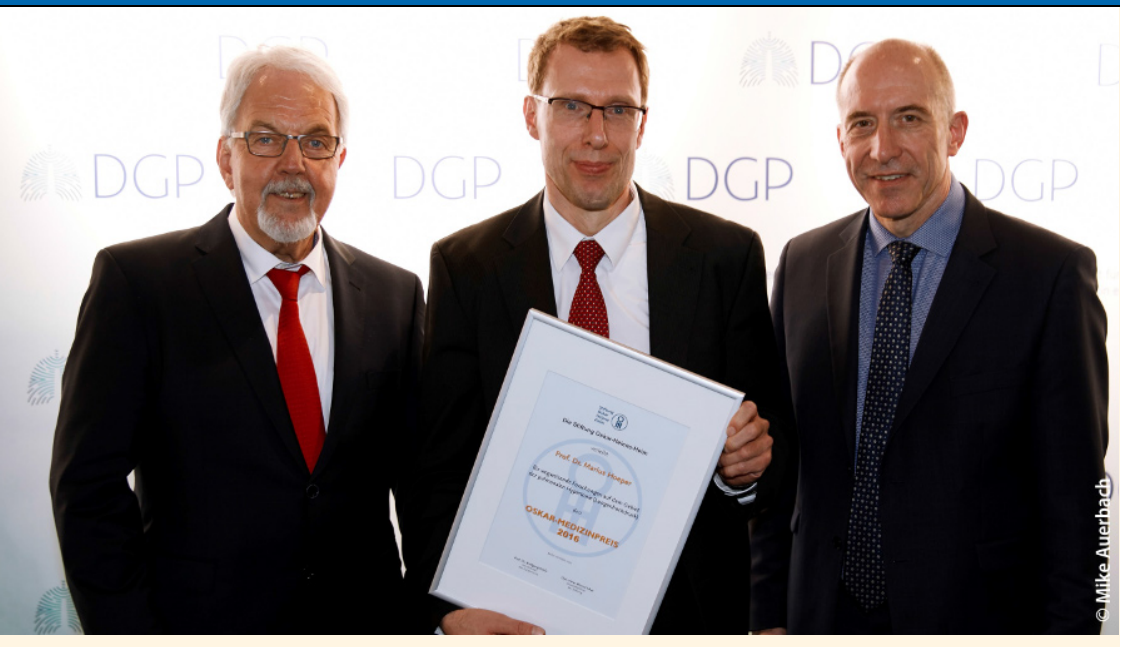

\section{Oskar-Medizinpreis 2016 zum Thema Pulmonale Hypertonie für Prof. Dr. Marius Hoeper}

Berliner Stiftung Oskar-Helene-Heim vergibt den Oskar-Medizinpreis 2016, dotiert mit 50.000 Euro, auf dem Gebiet der Pneumologie für Forschungsarbeiten zur Weiterentwicklung von Diagnostik und Behandlung von Erkrankungen des Lungengefäßsystems

Die pulmonale Hypertonie ist eine pathophysiologische Störung, die als Komplikation bei der Mehrzahl respiratorischer und kardiovaskulärer Krankheiten auftreten kann. Die Bezeichnung dient als Oberbegriff für verschiedenste Krankheitsbilder, die mit erhöhtem Blutdruck im Lungenkreislauf einhergehen. Es handelt sich um eine meist chronische Erkrankung, die im fortgeschrittenen Stadium als Folge von Rechtsherzversagen lebensbedrohlich sein kann. Die pulmonale Hypertonie wird oft sehr spät erkannt, da die Symptome anfangs nicht spezifisch sind. Frühzeitig erkannt, lässt sich die Erkrankung erfolgreich behandeln. Darauf sind die Forschungen von Prof. Hoeper ausgerichtet.

Mit der Verleihung an Prof. Hoeper würdigt die Stiftung die wissenschaftliche Qualität seiner 25-jährigen Forschungsarbeit, dokumentiert durch hochrangig publizierte Arbeiten und die Seniorautorschaft der aktuellen europäischen Leitlinie zur Diagnostik und Therapie der pulmonalen Hypertonie.

Das Preisgeld stellt die Stiftung für die Fortsetzung dieser Forschungen zur Verfügung.

Mitglieder der Jury für den Oskar-Medizinpreis 2016:

- Prof. Dr. Berthold Jany, Missionsärztliche Klinik Würzburg

- Prof. Dr. Horst Olschewski, Medizinische Universität Graz

- Prof. Dr. Klaus F. Rabe, LungenClinik Großhansdorf

- Prof. Dr. Silvia Ulrich Somaini, Universitätsspital Zürich

- Prof. Dr. Helmut Teschler, Ruhrlandklinik Essen

- Prof. Dr. Robert Loddenkemper, ehem. Lungenklinik Heckeshorn Berlin

Der Oskar-Medizinpreis 2016 wurde auf dem Kongress der Deutschen Gesellschaft für Pneumologie und Beatmungsmedizin am 23. März 2017 in Stuttgart verliehen.

Foto v.I.n.r. : Werner Ukas (Geschäftsführer Stiftung Oskar-Helene-Heim), Prof. Dr. Marius Hoeper (Medizinische Hochschule Hannover), Prof. Dr. Horst Olschewski (Medizinische Universität Graz)

Quelle:

Stiftung Oskar-Helene Heim, www.stiftung-ohh.de,

Geschäftsführer:WernerUkas, Kontakt:werner.ukas@stiftung-ohh.de 